\title{
Research on Deviation Force Demonstration Device Based on 3D Printing Technology
}

\author{
Mingsheng $\mathrm{Yu}^{1, \mathrm{a}}$, Chunyu Mao ${ }^{1, \mathrm{~b}^{*}}$, Xirui Sun ${ }^{1, \mathrm{c}}$ \\ 1 JILIN ENGINEERING NORMAL UNIVERSITY, Changchun, China \\ a599973567@qq.com, ${ }^{b} 290414003 @ q q . c o m,{ }^{c} 2441965204$ @qq.com
}

Keywords: Future education; STC12C5A60S2; Single-chip; Infrared photoelectric tube sensor

\begin{abstract}
With the development of the times, people pay more and more attention to the education of children, so education in the future is becoming more and more important, and in the future education also needs a lot of devices to enable students to understand the content of the teacher.

The demo device designed in this paper combines the mechanical structure and electrical circuit organically. Through the visual demonstration, it causes the students' curiosity. After the experiment and exploration, it deepens the impression and understanding of the bias force. The components required for the mechanical part are HYWB32212 standard bearings, the remaining base, the code plate and the guide are not the standard parts. We use the 3D printing technology. In the circuit control system, the main components are the intelligent controller (STC12C5A60S2 micro controller), the infrared photoelectric to Guan Chuan sensor module and the color LCD display module group. Yes. The mathematical model of the deflection force is set up. The angular velocity of the disc is detected by the infrared photo electronic pair of tube and the disc. The internal program is calculated by the single chip computer, and the display results are displayed on the display screen. The device works well and can play a great role in the future education and has a good market prospect.
\end{abstract}

\section{Introduction}

The design of the biasing force demonstration device is a device design for the educational demonstration in the "Future Education". Its purpose is to help the teachers to effectively teach in the classroom in the future education, and the students intuitively and effectively understand the bias. The deflection force demonstration device is designed to place a small ball on the inclined track. After releasing the small ball, it is converted into kinetic energy by the gravitational potential energy, and the small ball will move along the rotating orbit. If the rotating device is used as a reference frame, the ball has an initial velocity in the direction of the track. The track is in contact with the plate at the lowest point, where the ball enters the plane, at which point the ball has a tendency to move linearly. The ball is again in the rotary motion device. Here, we determine the direction of the Coriolis force direction and the angular velocity according to the right-handed spiral rule. First, the right hand is extended, and the four fingers other than the thumb are directed to the direction of the linear velocity of the non-inertial system, and then the four fingers are wound in the angular velocity direction[1]. At this point, the direction of the thumb is the Coriolis force direction. Then, the four fingers of the right hand except the thumb are bent inward in the direction of rotation, and the direction indicated by the thumb is the direction of the angular velocity, and the direction of the angular velocity is perpendicular to the plane of rotation[2]. The application of 3D printing technology is more and more extensive. We use a lot of 3D printing for this device. Many of the mechanical parts we need are printed by $3 \mathrm{D}$ printing[3].

\section{Mechanical Structure Design.}

Code disk design. A code wheel is a digital encoder for measuring angular displacement. It has the advantages of high precision, high resolution and high reliability. It is a commonly used displacement sensor that measures the angular position of the shaft. The code wheel is an absolute encoder. . Use 
CATIA software to draw the shape of the code wheel, then edit the parameters needed in the 3D printer through Cura DGO_14.07.01 software, and print the code disk through the 3D printer. The function of the code wheel here is to measure the ball. Speed and angular velocity[4].
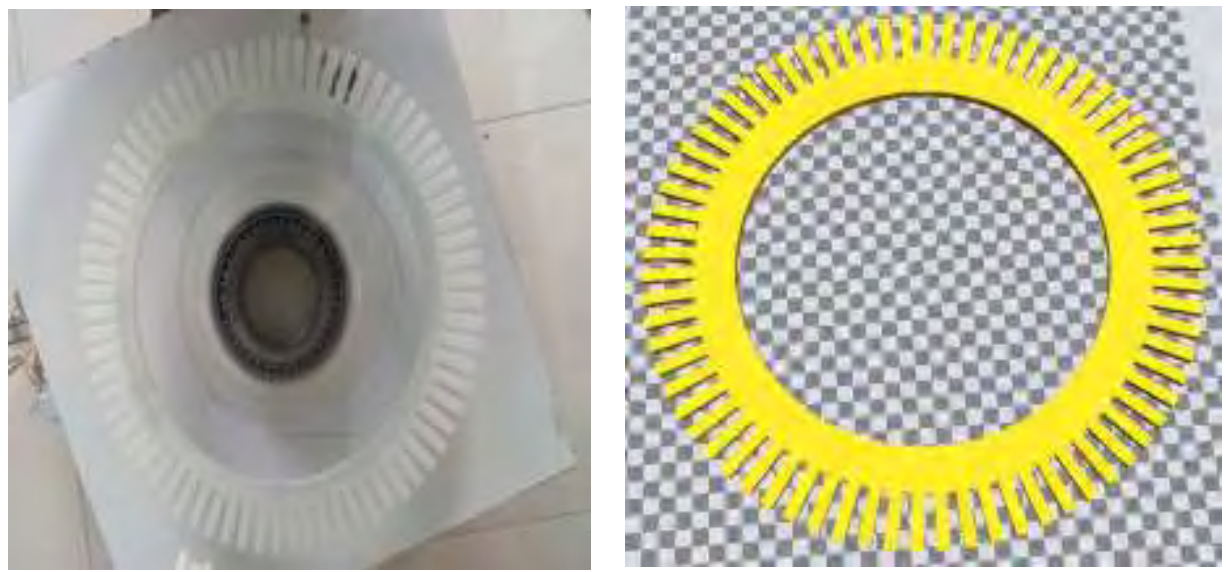

Fig 1. Code shape chart

3D molding process. In this design, many of the parts we need are printed by 3D printer. Through this design, we use 3D printing technology to let us know more about the convenience of 3D printing technology. Let's take a look at 3D printers and Cura DGO software for processing 3D printer data[5]. Cura DGO software parameter settings.As shown in the figure, the interface of cura software is very simple. The title bar consists of five parts: files, tools, machines, advanced options, and help. The commonly used commands are files and tools, and the following basic and advanced. Plug-ins, start/stop G Code These four are commonly used commands for parameter settings.

The following figure shows two common pages for parameter settings. The basic data of the parameters are also as shown:
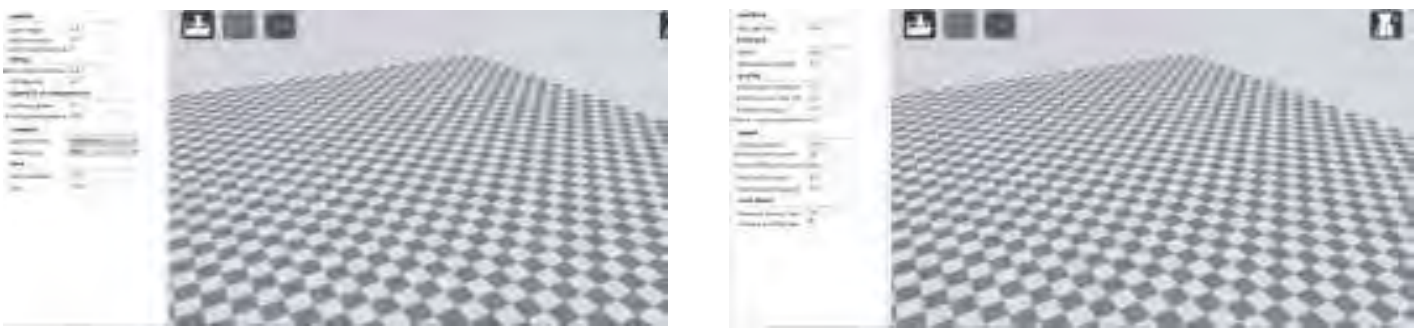

Fig 2.cura software parameter interface

Track design. The track here is printed on a 3D printer. We place the ball on the top of the track. When we give a force, the ball slides down the track and rotates into the tempered glass plane below.

As shown in the figure, first draw a 3D map of a code wheel with CATIA software, export the stl format, and open the stl file with the cure software. Here we set the basic parameters and the parameters printed on the previous code wheel are the same, no need to change here. For advanced settings, we can also follow the original data processing. If you need better part density, you can set the parameters more precisely, but because this part is slightly smaller, the printing time will be reduced, and printing will be needed. 6 hours. 


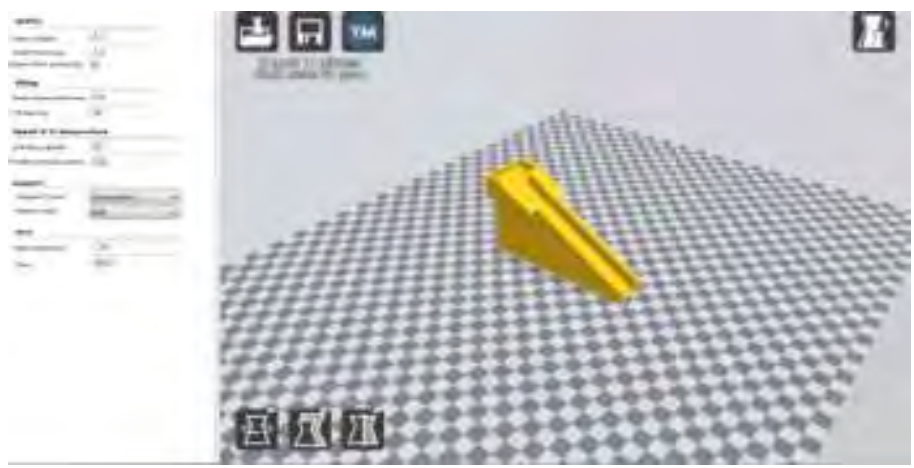

Fig 3. 3D drawings of track printing

base design. When designing the base, we have to consider putting the tapered roller bearing in. The device does not rub against the base when it is rotated, and the base is also large enough to not deflect the entire device. I use CATIA software to draw the 3D image as shown in the figure, save it as stl format, use the cure software to open the stl file. Here we set the basic parameters and the parameters printed on the previous code wheel are the same, no need to change here. For the initial settings in the advanced settings, the first two prints are $0.3 \mathrm{~mm}$, where the initial stack height is set to $0.2 \mathrm{~mm}$. The parameters in the rest of the advanced settings are processed according to the original data. If you need better part density, you can set the parameters more precisely, but because this part is slightly smaller, the printing time will be reduced. It takes about 17 hours.
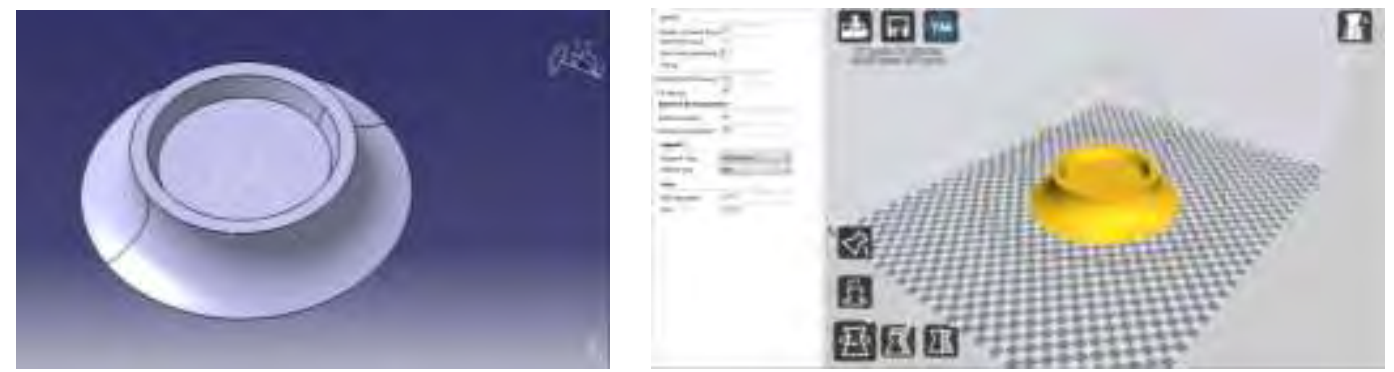

Fig 4. Three-dimensional shape of the base

Plans and models. According to the above design, the scheme obtained by CATIA drawing is as follows (slightly omitted):

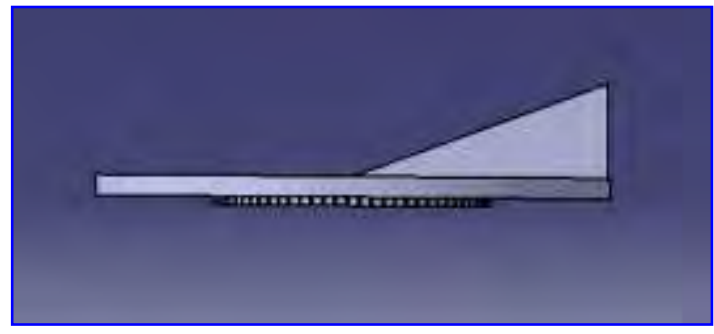

Fig 5. Main view

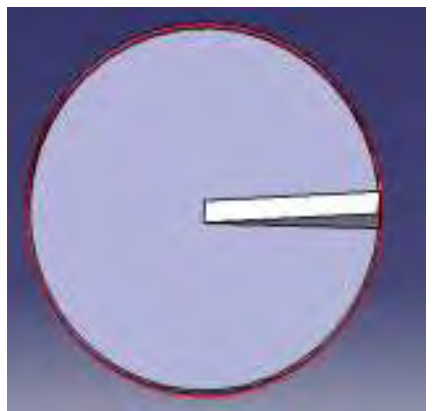

Fig 6. Top view

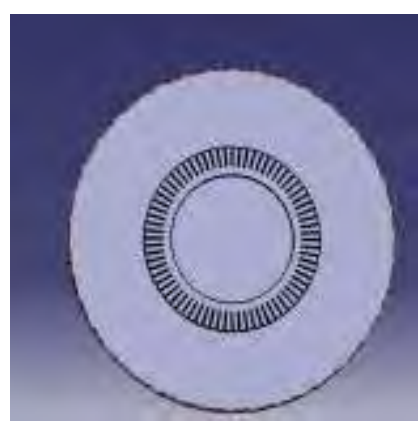

Fig 7.Bottom view 
Through the design, processing and debugging, the corresponding exhibits were produced and successfully realized. Fig 8 shows the physical diagram of the device, in which the diameter of the ball is $14 \mathrm{~mm}$, the mass is $11.3 \mathrm{~g}$, the height of the guide rail is $60 \mathrm{~mm}$, and the friction of the guide rail is $0.678 \mathrm{~J}$.

When the ball is released, it will enter the plane along the guide rail, and will be affected by the Coriolis force on the rotating plane, and will follow an arc-shaped trajectory. We measured the angular velocity of rotation through the microcontroller and the sensor, and calculated the Coriolis force from the above data and displayed it on the screen.

Mathematical Derivation. The small ball enters the plane state diagram as shown in Fig 8:

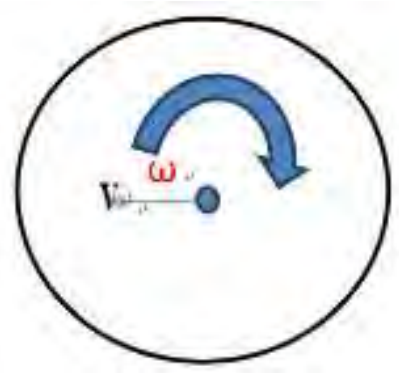

Fig 8. Small ball entering the plane state diagram

Let the mass of the ball be $\mathrm{m}$, the vertical height of the ball and the horizontal plane be $\mathrm{h}$, $\mathrm{v}^{\prime}$ is the velocity of motion (vector) relative to the rotating reference point, $\omega$ is the angular velocity (vector) of the rotating system, and $\times$ indicates the outside of the two vectors. Product symbol ( $\omega \times \mathrm{v}^{\prime}$ : the size is equal to the size of $\omega$ multiplied by the size of $v$ and multiplied by the sine of the angle between the two vectors, the direction meets the right-handed screw rule).

Then there is: $\mathrm{F}=-2 \mathrm{~m} \omega \times \mathrm{v}^{\prime}$ (Coriolis force expression formula)

First of all:

Gravity potential energy:

Friction consumes energy:

$$
\mathrm{Ep}=\mathrm{mgh}
$$

$$
\mathrm{w}=\mathrm{fs}
$$

When the ball is at the highest point of the orbit, it has the greatest gravitational potential energy.

When the ball moves along the orbit to the lowest point:

$\mathrm{v}^{\prime}=\sqrt{ }(2 \mathrm{gh}-2 \mathrm{fs} / \mathrm{m})$

It is not difficult to see here that the higher the angular velocity is, the faster the rotational speed of the disk is, and the greater the force on the ball. However, as a vector, force can be calculated here. Let's judge the direction of force.

According to the right hand spiral rule. First, the right hand is extended, and the four fingers other than the thumb are directed to the direction of the linear velocity of the non-inertial system, and then the four fingers are wound in the angular velocity direction. At this point, the direction of the thumb is the Coriolis force direction. The direction of the angular velocity also requires a right-hand rule: the finger of the right hand except the thumb is bent inward in the direction of the rotation, and the direction indicated by the thumb is the direction of the angular velocity, that is, the angular velocity direction is perpendicular to the plane of rotation[5]. Fig 9 shows the right-handed screw rule used by Coriolis force.The force diagram shown below is shown in Fig 10.

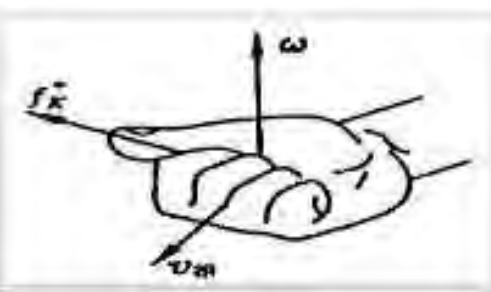

Fig 9 The right-handed screw rule

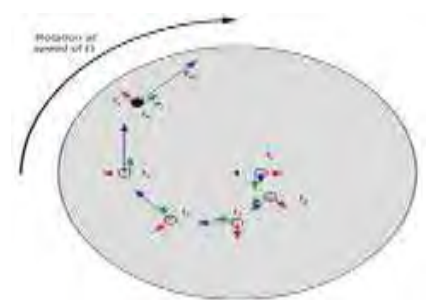

Fig 10 Force diagram 


\section{Electronic Control System Design.}

Control system design The one-piece minimum system board, the single-chip microcomputer used here is STC12C5A60S2. This system board is more convenient to operate, and it can also be directly connected with our LCD color screen display[6].

Color screen LCD display design Color screen LCD display features: new high quality; high quality LCD replacement parts; it is used to repair faulty screens: display problems, dead pixels, cracked LCD screen, wrong color issues; highly recommended professional installation; specification: Dimensions: 1.44 inches shielding material: thin film transistor; resolution: 128X128; driver chip: ST735R; Interface mode: 4-wire SPI serial bus; module pin: 11PIN; module size: $31.49 \mathrm{~mm}$ * 43.95mm[7-9].

Infrared photoelectric tube design. Features of infrared photoelectric tube: Built-in anti-jamming chip, strong anti-interference, more accurate measurement object; and built-in imported chip, the reaction is more sensitive, and the sensitivity is adjustable, the knob is simple and convenient, generally only $2.5 \mathrm{~ms}$ from detecting the object to reacting, which is called fast; The LED bulbs set therein have a longer service life; short-circuit protection, reject failure: the power supply is fully short-circuit protected, and the measurement is safe and worry-free[10].

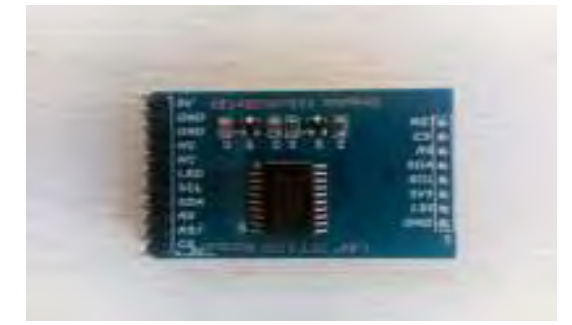

Fig 11. Color LCD display

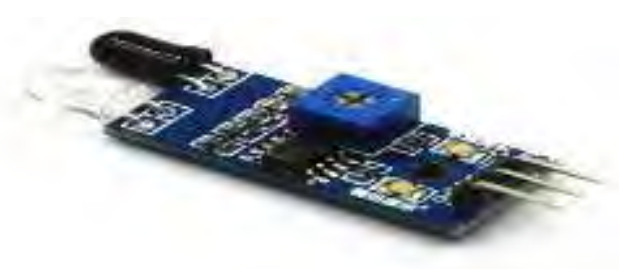

Fig 12. Infrared photoelectric tube

\section{Summary}

This exhibit perfectly designs the mechanical structure, and uses 3D printing, sensor technology and single-chip technology to complete the production and debugging of the exhibits. It is an exhibit that combines the mechanical and electronic experience to experience the bias. The audience can observe the small ball movement track and the first time. The different phenomenon in the imagination, that is, the small ball does not roll along the line, attracting the attention of the audience, and then exploring whether there is a physical law that is inconsistent with common sense leads to the above phenomenon, and then observes the angular velocity of the display and the trajectory of the small ball. The relationship allows people to further think about the relationship between Coriolis force and angular velocity. The works are well-made, and many popular technologies are applied. The phenomenon of bias is well displayed and will surely shine in future education.

\section{Acknowledgments}

Thanks to Mr. Chunyu Mao for his careful guidance on this paper. This research was supported by Program for Innovative Research Team of Jilin Engineering Normal University.

\section{References}

[1]Jianzhuang Wang, Bo Wu, Guoxu Liu, Tianzhao Bu, Tong Guo, Yaokun Pang, Xianpeng Fu, Junqing Zhao, Fengben Xi,Chi Zhang. Flexure hinges based triboelectric nanogenerator by 3D printing [J]. Extreme Mechanics Letters, 2018, 20 
[2]Yani Jiang,Jiping Zhou,Zhe Yang,Dongfang Liu,Xiaodong Xv,Guoqi Zhao,Hongcan Shi,Qi Zhang. Dialdehyde cellulose nanocrystal/gelatin hydrogel optimized for 3D printing applications[J]. Journal of Materials Science,2018,53(16).

[3]Jin Woo Jung,Hyungseok Lee,Jung Min Hong,Jeong Hun Park,Jung Hee Shim,Tae Hyun Choi,Dong-Woo Cho. A new method of fabricating a blend scaffold using an indirect three-dimensional printing technique[J]. Biofabrication,2015,7(4).

[4]Jamison Go,A. John Hart. A framework for teaching the fundamentals of additive manufacturing and enabling rapid innovation[J]. Additive Manufacturing,2016,10.

[5]J. Czyżewski, P. Burzyński, K. Gaweł, et al. Rapid prototyping of electrically conductive components using 3D printing technology[J]. Journal of Materials Processing Tech, 2009, 209(12):5281-5285.

[7]Jing Ping Yuan,Fen Fen Yu. Design of a Fast Resistance Sorting Instrument Based on STC Microcontroller[J]. Applied Mechanics and Materials,2014,3360(599).

[8]Li Gang Chen,Xue Feng Yang,Bo Qu. Design of Intelligent Locomotive Based on STC12C5A60S2[J]. Advanced Materials Research,2014,3137(912).

[9]Kyung Joon Kwon,Min Beom Kim,Cheon Heo,Seong Gyun Kim,Jong Sang Baek,Young Hwan Kim. Wide color gamut and high dynamic range displays using RGBW LCDs[J]. Displays,2015,40.

[10]M S Khan,F M Pathan,Prof U V Shah,Prof D H Makwana,Prof B G Anandarao. Development of CCD controller for scientific application[J]. Journal of Physics: Conference Series,2010,208(1). 